\title{
Can we evaluate cranial aneurysms on conventional brain magnetic resonance imaging?
}

\author{
Emine Caliskan, Yeliz Pekcevik ${ }^{1}$, Adnan Kaya ${ }^{2}$ \\ Departments of Radiology and ${ }^{2}$ Cardiology, Suruc State Hospital, Sanliurfa, ${ }^{1}$ Department of Radiology, Izmir Tepecik Training and Research \\ Hospital, Izmir, Turkey
}

\begin{abstract}
Purpose: To evaluate the contribution of conventional brain magnetic resonance imaging (MRI) for the determination of intracranial aneurysms. Materials and Methods: Brain MRI and computed tomography angiography (CTA) of 45 patients ( 29 women and 16 men; age range, 32-80 years) with aneurysm were analyzed. A comparison was made between brain MRI and CTA based on size and presence of aneurysm. The comparisons between MRI and CTA were investigated through Bland-Altman graphics, receiver operating characteristic curve, and Kappa statistics. Results: Fifty-seven aneurysms were evaluated. Forty-five percent of 57 aneurysms on CTA were detected on conventional brain MRI. A significant correlation was found between CTA and brain MRI in the diagnosis of aneurysm $(P<0.05)$. In an analysis of the size measurement, a significant correlation was observed between CTA and brain MRI. Seventy-seven percent of aneurysms $<4 \mathrm{~mm}$ was not detected and the efficiency of MRI in the detection of aneurysms $<4 \mathrm{~mm}$ was found to be low. Conclusion: Aneurysms can also be appreciated on conventional brain MRI, and vascular structures should be reviewed carefully while analyzing brain MRI.
\end{abstract}

Key words: Computed tomography angiography, intracranial aneurysm, magnetic resonance imaging

\section{Introduction}

Brain magnetic resonance imaging (MRI) has become a modality, used frequently and with a growing demand in the diagnosis of many diseases. Tumor, neuro-degenerative diseases, intracranial hemorrhage, and infection are some of these diseases and are mainly related to brain parenchyma. Magnetic resonance angiography (MRA), computed tomography angiography (CTA), and digital subtraction angiography (DSA) are known as imaging methods preferred in cranial vascular pathologies. ${ }^{[1,2]}$ These angiographic methods really provide results with a high accuracy in the diagnosis of aneurysm. Each of these methods has advantages and disadvantages over each other ${ }^{[1-7]}$ Although, DSA is still accepted as the gold standard method of detecting intracranial aneurysms,

Address for correspondence:

Dr. Adnan Kaya, Department of Cardiology,

Suruc State Hospital, Sanliurfa, Turkey.

E-mail: adnankaya@ymail.com

\begin{tabular}{|l|l|}
\hline \multicolumn{2}{|c|}{ Access this article online } \\
\hline Quick Response Code: & Website: \\
\hline & www.ruralneuropractice.com \\
\cline { 2 - 2 } & \\
\hline
\end{tabular}

CTA is cheaper, easily accessible, safer, and particularly noninvasive when compared to DSA. CTA has become a widely used imaging method, which provides highly accurate results and has gained acceptance in the diagnosis of vascular diseases. This is a useful and valuable method providing results with a similar accuracy in the diagnosis of aneurysm when compared to DSA. ${ }^{[1,7]}$

In the routine practice, we encountered more brain MRIs than MRA and CTA. Moreover, in this study, we aim to evaluate the contribution of conventional brain MRI in the diagnosis of intracranial aneurysms.

\section{Materials and Methods}

\section{Patients}

Between June 2012 and April 2014, CTA of 45 patients (29 women and 16 men; age range, 32-80 years) with 57 intracranial aneurysms were

This is an open access article distributed under the terms of the Creative Commons Attribution-NonCommercial-ShareAlike 3.0 License, which allows others to remix, tweak, and build upon the work non-commercially, as long as the author is credited and the new creations are licensed under the identical terms.

For reprints contact: reprints@medknow.com

How to cite this article: Caliskan E, Pekcevik Y, Kaya A. Can we evaluate cranial aneurysms on conventional brain magnetic resonance imaging?. J Neurosci Rural Pract 2016;7:83-6. 
evaluated. The study included the patients that had initially been diagnosed with unruptured intracranial aneurysms on CTA and had brain MRI within 6 months before CTA. Conventional brain MRI of the patients that obtained before CTA was reviewed retrospectively by two radiologists who were blind to the CTA diagnosis.

\section{Computed tomography angiography}

All computed tomography (CT) examinations were performed by a 64-slice CT scanner (Aquilion 64, Toshiba Medical Systems, Tokyo, Japan). For venous access, an upper extremity vein (antecubital vein of the right arm) and a 20-gauge IV cannula were used. After precontrast images a total of $80-85 \mathrm{~mL}$ of contrast media with high iodine concentration $(370-400 \mathrm{mg} / \mathrm{mL}$ ) was injected with a flow rate of $5 \mathrm{~mL} / \mathrm{s}$, followed by a $20 \mathrm{~mL}$ saline chaser. The scanning parameters included $120 \mathrm{kV}, 225 \mathrm{~mA}$, section thickness of $0.5 \mathrm{~mm}$, and reconstruction interval of $0.3 \mathrm{~mm}$. The scan revolution time was $0.4 \mathrm{~s}$. Data for CTA were obtained in a caudocranial direction. All multidetector $\mathrm{CT}$ angiography data were transferred from the archive to a workstation (Aquarius workstation, TeraRecon, San Mateo, California, USA), via internal network connections, providing three-dimensional (3D) postprocessing options, multiplanar image reformatting, and maximum intensity projections.

Aneurysms were investigated in terms of number and size. The aneurysm with the largest dimension was taken as a reference in 3D images for size measurement.

\section{Magnetic resonance imaging}

All studies were performed using a 1.5 T system (Achieva; Philips Medical Systems, Best, The Netherlands) using T1-weighted (T1W) sagittal spin-echo (repetition time [TR]: $500 \mathrm{~ms}$; echo time [TE]: $15 \mathrm{~ms}), \mathrm{T} 2 \mathrm{~W}$ transverse fast spin-echo (TR: $3.300 \mathrm{~ms}$; TE: $100 \mathrm{~ms}$ ), and fluid-attenuated inversion recovery coronal (TR: $8000 \mathrm{~ms}$; TE: $110 \mathrm{~ms}$; T1: $2400 \mathrm{~ms}$ ) sequences.

The evaluation of the aneurysms was made based on detection of focal dilatation by using axial $\mathrm{T} 2 \mathrm{~W}$ images as the main sequence. The largest dimension on axial $\mathrm{T} 2 \mathrm{~W}$ images was taken into consideration for the measurements.

\section{Statistical analysis}

Mean, standard deviation, median, maximum and minimum rate, and frequency values were used in the defining statistics of the data. The correlation between the measurements was determined by using intraclass correlation analysis. The difference distribution between measurements was shown in Bland-Altman graphics. Aneurysm size of the patients with a positive
CTA but a negative MRI was analyzed through receiver operating characteristic curve. Kappa test was used for the agreement analysis. SPSS 22.0 (SPSS Inc., Chicago, Illinois, USA) was used for statistical analysis.

\section{Results}

Examination of the remaining 45 patients revealed the presence of 57 aneurysms on CTA. Seven patients had two aneurysms; one patient had three aneurysms and one patient had four aneurysms. Forty-five percent (45 aneurysms) of 57 aneurysms were detected on brain MRI. 16-45 detected aneurysms were found in middle cerebral artery bifurcation, 12 in distal internal carotid artery, 9 in anterior communicating artery, and 8 in the basilar artery [Figures 1 and 2]. Significant correlation was found between CTA and brain MRI $(P<0.05)$. Sensitivity, positive predictive value, specificity, and negative predictive value were $79 \%, 100 \%, 100 \%$, and $29 \%$, respectively.

Twelve aneurysms out of 57 were not detected on brain MRI. Aneurysm size on CTA had a differential effect for 12 aneurysms which was found negative on MRI. Forty-five aneurysms were appreciated on brain MRI (area under the curve [AUC]: 0.918 [0.820-1.00]/ $P<0.001)$. The rate of detecting aneurysms $<4 \mathrm{~mm}$ on CTA as negative on MRI was $77 \%$ and had a significant effect (AUC: $0.883[0.752-1.00] / P<0.001)$.

The sizes of aneurysms varied between $1.5 \mathrm{~mm}$ and $25.5 \mathrm{~mm}$ on CTA (median size: $7.2 \pm 4.1 \mathrm{~mm}$ ) and between $2 \mathrm{~mm}$ and $25 \mathrm{~mm}$ on brain MRI (median size: $7.2 \pm 4.0 \mathrm{~mm}$ ). There was a significant correlation on the size measurement of aneurysms between CTA and brain MRI $(r=0.959[0.927-0.977] / P<0.001)$ [Figure 3]. The

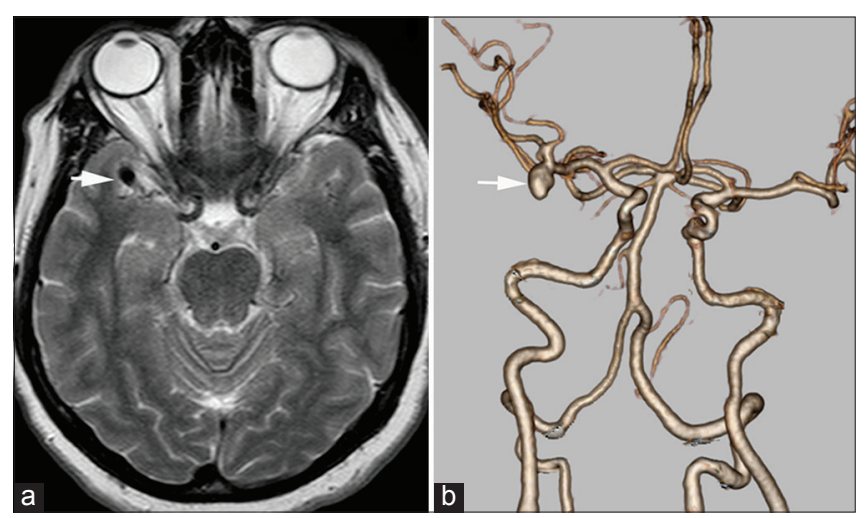

Figure 1: Forty-six-year-old female with right middle cerebral artery aneurysm. (a) T2-weighted axial image shows an abnormal signal void in the right Sylvian fissure (arrow) (b) computed tomography angiography three-dimensional volume rendered image demonstrates the right middle cerebral artery bifurcation aneurysm (arrow) 


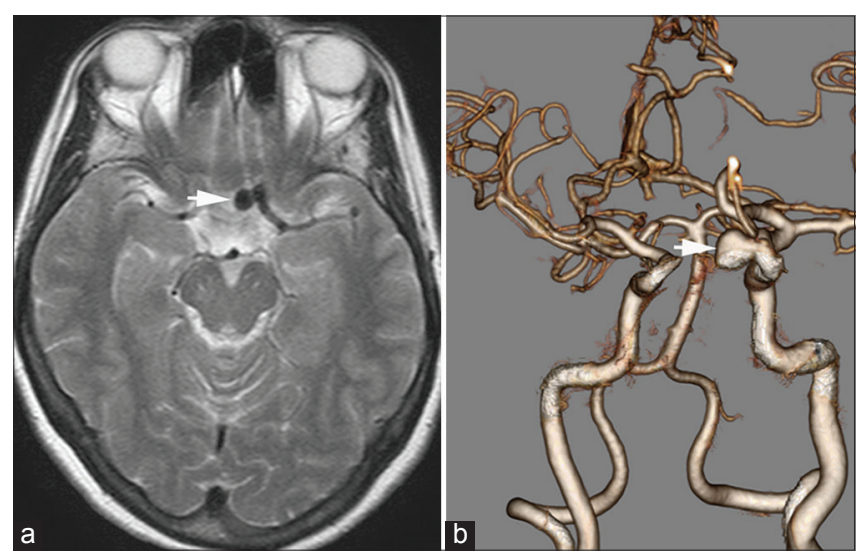

Figure 2: Forty-nine-year-old female with left internal carotid artery aneurysm. (a) T2-weighted axial image shows an abnormal signal void in the left suprasellar cistern (arrow) (b) computed tomography angiography three-dimensional volume rendered image demonstrates aneurysm in the left internal carotid artery cavernous segment (arrow). The aneurysm is measured bigger in size on computed tomography angiography

mean difference between CTA and MRI measurements was $0.8 \mathrm{~mm}$. The maximum difference was $5.4 \mathrm{~mm}$. There was no aneurysm detected greater in size on brain MRI. All aneurysms were detected less in size than CTA on brain MRI.

\section{Discussion}

This study demonstrates a significant correlation between brain MRI and CTA in the detection of intracranial aneurysm. Nearly half of the aneurysms that were detected on CTA were also appreciated on brain MRI. Conventional routine brain MRI, which is highly substantial in the diagnosis of many parenchymal diseases and does not use ionizing radiation, is also valuable in the diagnosis of vascular diseases. This characteristic of brain MRI could be considerably because more careful approaches toward vascular structures on a conventional brain MRI scanned in daily practice for any reason may increase the chance of detecting an asymptomatic aneurysm. Moreover, aneurysm, which is found incidentally and stands out with its focal dilatation, may provide a possibility for treatment with an early diagnosis.

Although 45 aneurysms of all 57 aneurysms on CTA were detected on brain MRI, the fact that the rest 12 aneurysms were not detected brings size correlation into mind. Seventy-seven percent of aneurysms $<4 \mathrm{~mm}$ could not be detected on conventional brain MRI. In other words, efficiency of conventional brain MRI in detecting aneurysms below $4 \mathrm{~mm}$ was found low. But, it is known that aneurysm more likely to rupture

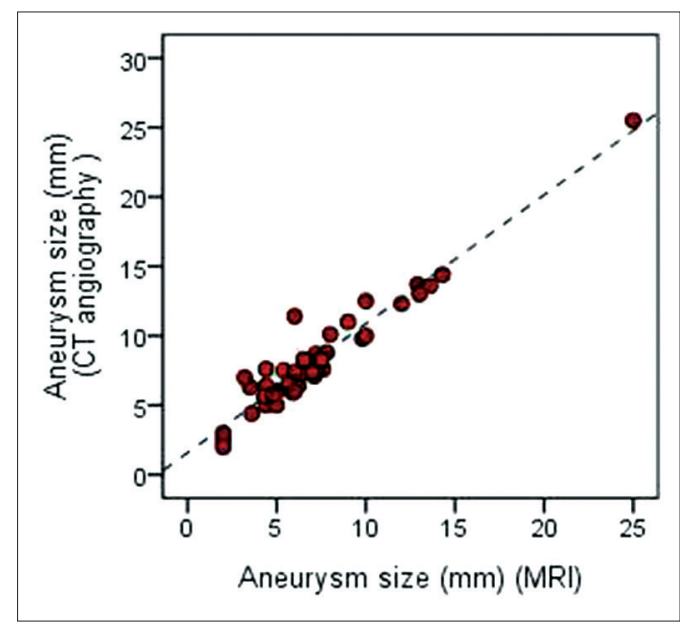

Figure 3: Although, the aneurysms were measured bigger in size on computed tomography angiography when compared with brain magnetic resonance imaging, measurement of the intracranial aneurysms' size showed correlation between computed tomography angiography and brain magnetic resonance imaging. The graphic shows this correlation

when it is more than $7 \mathrm{~mm} \cdot{ }^{[8]}$ Ruptured aneurysm and intracranial hemorrhage are closely related to mortality and morbidity. ${ }^{[8]}$

We also found significant correlation when brain MRI and CTA were compared in terms of size measurement. All aneurysms were detected on brain MRI with a smaller size, but the sizes were close to sizes measured on CTA (maximum difference: $5.4 \mathrm{~mm}$, median difference: $0.8 \mathrm{~mm})$.

There are some limitations in our study. The numbers of patients were small. The major reason for that was we worked on patients diagnosed with unruptured asymptomatic intracranial aneurysms on CTA and who had brain MRI before CTA, within 6 months. Additionally, investigating the role of aneurysms' localization, morphology (saccular, fusiform, etc.), and the role of the thrombosis in detecting the presence of aneurysm can be guiding for further studies.

\section{Conclusion}

Comparisons of angiographic methods such as DSA, CTA, and MRA were performed in literature several times in the diagnosis of aneurysms. ${ }^{[1-3,5-10]}$ As far as we know, a comparison like the one in this study between conventional brain MRI and CTA has not been performed. Aneurysms, especially larger than $4 \mathrm{~mm}$, can be appreciated on conventional brain MRI when the vessels carefully investigated. 


\section{Financial support and sponsorship}

Nil.

\section{Conflicts of interest \\ There are no conflicts of interest.}

\section{References}

1. Kumar A, Kato Y, Motoharu H, Sifang C, Junpei O, Takeya W, et al. An update on three-dimensional CT angiography in aneurysms: A useful modality for a neurosurgeon. Turk Neurosurg 2013;23:304-11.

2. Miloševic Medenica S, V Vuckovic V, Prstojevic B. 64-Slice CT angiography in the detection of intracranial aneurysms: Comparison with DSA and surgical findings. Neuroradiol J 2010;23:55-61.

3. Delgado Almandoz JE, Jagadeesan BD, Refai D, Moran CJ, Cross DT $3^{\text {rd }}$, Chicoine MR, et al. Diagnostic yield of computed tomography angiography and magnetic resonance angiography in patients with catheter angiography-negative subarachnoid hemorrhage. J Neurosurg 2012;117:309-15.

4. Numminen J, Tarkiainen A, Niemelä M, Porras M, Hernesniemi J, Kangasniemi M. Detection of unruptured cerebral artery aneurysms by MRA at 3.0 tesla: Comparison with multislice helical computed tomographic angiography. Acta Radiol 2011;52:670-4.

5. Prestigiacomo CJ, Sabit A, He W, Jethwa P, Gandhi C, Russin J. Three dimensional CT angiography versus digital subtraction angiography in the detection of intracranial aneurysms in subarachnoid hemorrhage. J Neurointerv Surg 2010;2:385-9.

6. Yanamadala V, Sheth SA, Walcott BP, Buchbinder BR, Buckley D, Ogilvy CS. Non-contrast 3D time-of-flight magnetic resonance angiography for visualization of intracranial aneurysms in patients with absolute contraindications to CT or MRI contrast. J Clin Neurosci 2013;20:1122-6

7. Yoon DY, Lim KJ, Choi CS, Cho BM, Oh SM, Chang SK. Detection and characterization of intracranial aneurysms with 16-channel multidetector row CT angiography: A prospective comparison of volume-rendered images and digital subtraction angiography. AJNR Am J Neuroradiol 2007;28:60-7

8. Villablanca JP, Duckwiler GR, Jahan R, Tateshima S, Martin NA, Frazee J, et al. Natural history of asymptomatic unruptured cerebral aneurysms evaluated at CT angiography: Growth and rupture incidence and correlation with epidemiologic risk factors. Radiology 2013;269:258-65.

9. Ramasundara S, Mitchell PJ, Dowling RJ. Bone subtraction CT angiography for the detection of intracranial aneurysms. J Med Imaging Radiat Oncol 2010;54:526-33.

10. Roth C. Value of CT and MR angiography for diagnostics of intracranial aneurysms. Radiologe 2011;51:106-12. 